which can be made arbitrarily small by ehoosing $N$ large enough. This proves Wrdös' theorem for $f(p) \neq f(q) \cdot(f(p) \neq 0, f(q) \neq 0)$. If for some sequence $f\left(p_{1}\right)=f\left(p_{2}\right)=\ldots$, then, considering the expression

instead of

$$
\sum_{f(p)=y_{l}}\left(\cos g_{l} T y-1\right) \sum_{f(p)=y_{l}} \frac{1}{p}
$$

$$
\sum \frac{\cos f(p) x y-1}{p}
$$

one can repeat the argument above and our statement follows again.

\title{
References
}

[1] P. Erdös, On the density of some sequences of numbers ITI, J. Loudon Math. Soc. 13 (1938), pp. 119-127.

[2] P. Erdös and A. Wintner, Additive arithmelical funotions and statistical inde. pendence, Amer. J. Math. 61(1939), pp. 713-721.

[3.] M. Kac, Statistical independence in probability, analysis and number theory, Carus Matik. Monographs, 1964.

[4] A. Rényi, On the distribution of values of additive number-theoretical fundtions, Publ. Math. 10 (1963), pp. 264-273.

[5] - Probabitity Theory, Amstcrdam-London 1970.

[6] I. J. Schoenberg, On asymptotic distributions of arithmetio functions, Trans. Amor. Math. Soo. 39 (1936), pp. 315-330.

MATA. DEPT. SUNY AT STONY BROOK

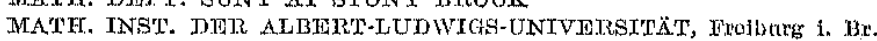

\section{Some remarks on the decomposition of a rational prime in a Galois extension}

by

M. Braskanan (Perth, W. Australia)

1. Inderaduction. Not much is known abont the lith of decomposition of rational primes in a Galois extension if the extension is not abelian. It is known that only for abelian extensions wo ean give a simple law of decomposition depending on the residne of the given prime with respect to a certain unodulvs. The object of the present paper is to get some information about the relationship between the number of prime divisors of a given rational prime and a rational prime which is ramitied in a Galois extension. This information also holps us to get some idea abont the class numbers of certain algobrato number fields. For example, the well-known result that the alass number of the field $Q\left(v^{r} a\right)$ (r odd prime and $a$ is divisible by a prime of the form $m+1$ ) js divisible by $r$ could be deduced from our resulti.

I wonld like to thank Professor A. Schinzel and the referee for their valuable commentis in the preparation of the paper.

2. Notations and preliminaries. Throughont this paper, $Q$ denotes the rational number fidd, 7 denotes a finite Galois axtension of $Q$ with Galois group $a$ and $\sigma_{k}$ denotes the ring of integers of 7 . The prime ideals of $\mathcal{O}_{k}$ aro called $k$ primes. $y$ and $q$ denote distinct rational primes and $P$ and $\mathfrak{Q}$ denote the $h$-primes lying above $p$ and $q$ respoctively. $g_{l}$ denotes the number of distinet k-primes \& lying above the rational prime $l . \theta_{b}$ and fi denoto the rarnification index. and residue class degree respectively

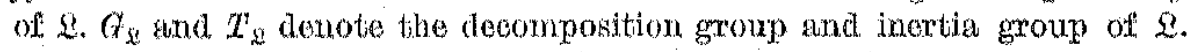
They are subgroups of $Q$ of order $e_{l} f_{l}$ and $e_{l}$ respectively. $T_{2}$ is a stakgroup of $G_{g}$ and its elements induce the trivial automorphism on the residue class field of $S_{2} g_{l}$ will be the number of cosets of $Q_{2}$ in $Q$. Let $Q=\bigcup_{j=1}^{g / b} \tau_{j} Q_{s}$ be a coset decomposition of $G_{\Omega}$ in $G$. Then the k-primes $\tau_{j} \mathfrak{Q}$ are precisely the distinet $k$-primes lying above $l$, 
If $x$ is the smallest positive integer such that $q^{x}=1 \bmod p$, then we say that $x$ is the order of $q$ with respect to $p$ and it is denoted by ord $q$. $(a, b, c, \ldots)$ denotes the G.C.T. of $a, b, c, \ldots, a \mid b$ means $a$ divides $b, a+b$ means $a$ does not divide $b$ and $a^{w} \| b$ means $a^{2 w} \mid b$ but $a^{a+1} \nmid b$.

3. Main results. We first prove the following

THwonewr 1. Let $(k: Q)=n$ and $e$ be a positive integer such that $(e, n / e)$ $=1$ and $e \mid\left(g_{q}, e_{p}, p-1\right)$. Then if $q$ splits into principal k-primes,

$$
e \mid e(p-1) / \operatorname{ord}_{p} q
$$

where

$$
c= \begin{cases}1 & \text { if } e \text { is odd or } p \equiv 1 \bmod 2, \\ 2 & \text { otherwise. }\end{cases}
$$

Proof. If $e=1$, there is nothing to prove. So let us assume $e>1$. Let $u$ be a prime factor of $e$ and $u^{t} \| e$. Without loss of generality, we prove the theorem when $e$ is replaced by $u^{t}$. Take any Sylow $u$-subgroup $F$ of $T_{\mathfrak{P}}$ which is of order $u^{t}$ since $\left\langle u^{l}, n / u^{t}\right\rangle=1$. The elements of $E$ belong to distinct cosets of $G_{52}$; for otherwise, if $\tau_{i}$ and $\tau_{j}$ of $\#$ belong to the same coset of $G_{2}$, then $\tau_{i} \tau_{3}^{-1} \in G_{Q}$ and so its order divides $n / u^{t}$ which is a contradiction. Let the elements of $\mathbb{E}$ be $\tau_{i}\left(i=1,2, \ldots, u^{t}\right), \tau_{1}$ being the identity of $G$.

Extend $E$ to a set $S$ consisting of elements in $G$ which represent the $g_{q}$ cosets of $G_{Q Q}$ in $G$. Let $\tau_{s}\left(s=1,2, \ldots, g_{q}\right)$ (the first $u^{t}$ elements being those of $E$ ) be the elements in $S$. Let the coset of $\tau_{s}$ be denoted by $\bar{\tau}_{s}$ and $\vec{S}$ be the set of these cosets. Now, we will arrange $g_{q}$ elements of $G$ which represent the distinct cosets in $g_{\alpha} / l^{t}$ columns in a suitable manner. For this, first put $\tau_{1}, \tau_{2}, \ldots, \tau_{u^{t}}$ in the first column. Take a $\tau_{i}$ from $S$ not belonging to the cosets $\bar{\tau}_{1}, \bar{\tau}_{2}, \ldots, \bar{\tau}_{u^{t}}$ and put $\tau_{1} \tau_{i}, \tau_{2} \tau_{i}, \ldots, \tau_{u^{l}} \tau_{i}$ in the second column. It is easy to see that the $2 u^{t}$ elements in these two columns belong to $2 u^{t}$ distinct cosets. Tale a $\tau_{j}$ from $S$ not belonging to the cosets of the $2 u^{i}$ elements already arranged. Put $\tau_{1} \tau_{j}, \tau_{2} \tau_{j}, \ldots, \tau_{u^{t}} \tau_{j}$ in the third column. We easily see that all the $3 u^{l}$ elements thus arranged belong to $3 u^{l}$ distinct cosets. Repeating this process $g_{q} / h^{t}$ times, we get the desired result. Thus, we get a set of $g_{\alpha}$ elements of $G$, which represent the $g_{\alpha}$ cosets in $\vec{S}$, in the form $\bigcup_{i=1}^{u^{t}} \tau_{i} F^{\prime}$ where $W^{\prime}$ consists of $g_{q} / u^{t}$ elements say $\sigma_{1}, \sigma_{2}, \ldots, \sigma_{q_{q} / u^{t}}$.

Now, let us assume that the 7 -primes lying above $q$ are principal and write the factorization of $(q)$ in the following manner:

$$
(q)=\prod_{i=1}^{q_{q}} \tau_{i} \mathfrak{Q}^{e_{\alpha}}=\prod_{j=1}^{u^{t}} \tau_{j}\left(\prod_{i=1}^{q_{q} / u^{l}} \sigma_{i} \mathfrak{Q}^{e_{\alpha}}\right)
$$

where $Q$ is a principal $k$-prime lying above $q$. Hence

$$
q=\varepsilon \prod_{j=1}^{u^{t}} \tau_{j}\left(\prod_{i=1}^{g_{q} / u^{t}} \sigma_{i} \gamma^{e} q\right)
$$

where $\gamma \in \mathscr{O}_{k}$ and generates $Q$ and $\varepsilon$ is a unit in $\mathscr{O}_{k}$ such that $\tau_{i}$ $\left(i=1,2, \ldots, g_{q}\right)$ fix $\varepsilon$. Applying $n / g_{q}$ automorphisms $v_{s}\left(s=1,2, \ldots, n / g_{q}\right)$ of $G_{Q}$ on both sides, we get

$$
g^{n / y_{2}}=\varepsilon^{\prime} \prod_{g=2 \times 1}^{n j g_{q}} v_{s}\left(\prod_{j=1}^{u^{t}} \tau_{j} \alpha\right)
$$

for some $a \in \mathcal{O}_{t b}$ and a unit $\varepsilon^{\prime}$ which remains fixed nnder all the automorphisms of $G$, i.e. $\varepsilon^{\prime}= \pm 1$.

Now

$$
\tau_{j} a=\alpha \bmod \mathfrak{P} \quad\left(j=1,2, \ldots, u^{2}\right)
$$

since $\tau_{j} \in T_{\mathfrak{F}}$ and so induces the trivial automorphism on the residue class field of $\mathfrak{B}$.

Hence

$$
\pm q^{n / q_{q}}=\alpha^{u^{l}} \bmod \mathfrak{m}
$$

Since $(e, n / e)=1$ and $e \mid g_{q}$, we have $\left(u^{t}, n / g_{q}\right)=1$. Then, it follows that

$$
\sharp q \equiv \beta^{u^{t}} \bmod \beta
$$

for some $\beta \epsilon \mathcal{O}_{k}$.

This shows that, if $u$ is odd or $p \equiv 1 \bmod 2^{t+3}, q$ is a $u^{t}$-th power modp. Otherwise, $q$ is a $w^{t} / 2$-th power modis.

Frence

$$
\operatorname{ord}_{p} q \mid\left(\frac{p^{f_{p}}-1}{u^{l}}, p-1\right)
$$

if $u$ is an odd prime or $p=1 \bmod 2^{t+1}$ and

otherwise.

$$
\left.\operatorname{ord}_{p} q\right)\left(\frac{p^{f_{p}}-1}{u^{1} / 2}, p-1\right)
$$

Now

and

$$
\left(p^{f_{p}}-1, p-1\right) \mid f_{p}(p-1)
$$

$$
\left(u^{t}, f_{p}\right)=1
$$

Consequently, we have

and

$$
u^{t} !(p-1) / \operatorname{ord}_{p} q \quad \text { if } u \text { is an odd prime or } p \equiv 1 \bmod 2^{t+1}
$$

$$
u^{t} \mid 2(p-1) / \text { ord } \mathrm{d}_{p} q \quad \text { otherwise. }
$$


Repeating our method for all other prime power factors of $e$ instead of $u^{t}$, we get our theorem.

When the class number of $k$ is relatively prime to $n$, we can delete the condition on $q$ that it splits into principal $k$-primes and state the theorem in the following manner:

THEOREM. $2 \cdot$ Let $(k: Q)=n$ and let the class number of $k$ be relatively prime to $n$. Let e be a positive integer such that

Then

$$
(e, n / e)=1 \quad \text { and } \quad e \mid\left(g_{a}, e_{p}, p-1\right)
$$

$$
e \mid c(p-1) / o r d_{p} q
$$

where $c=1$ if $e$ is odd or $p \equiv 1 \bmod 2 e$ and $o=2$ otherwise.

Proof. Let $K$ be the Hilbert class field of $k$ and let $(K: k)=h$. Then $(h, n)=1$ and $(K: Q)=n h$. Let $e_{l}^{K}$ and $g_{l}^{K}$ denote the ramification index of a $K$-prime lying above the rational prime $l$ and the number of distinct $K$-primes lying above $l$ respectively. Then, we can easily see that

and

$$
(e, n / e)=1 \quad \text { implies } \quad(e, n h / e)=1
$$

$$
e \mid\left(g_{q}, e_{y}, p-1\right) \quad \text { implies } e \mid\left(g_{q}^{K}, e_{p}^{K}, p-1\right) .
$$

Taking $K$ for $k$ in Theorem 1 , we see that $e$ satisfies the required conditions and so the theorem follows since every $k$-prime splits into principal $K$-primes.

\section{Arithmetic euclidean rings}

by

OcxFourd Qumin (Bethlehem, Ponn.)

1. Introduction. Jet $A$ be an integral domain. We shall say that $A$ is a euctidean ring, or simply $A$ is euclidean, if there exists a map $\varphi$ : $A-\{0\} \rightarrow N, N$ the non-negative integers, satisfying the following two properties:

1.) If $a, b \in A-\{0\}$, then $p(a b) \geqslant \varphi(a)$;

2) If $a, b \in A, b \neq 0$, then there exist $q, r \in A$ such that $a=b q+r$, where $r=0$ or $\varphi(r)<\varphi(b)$.

It is easy to see that condition 1 ) is an unnecessary restriction; i.e., if there is a map $\varphi: A-\{0\} \rightarrow N$ satisfying only condition 2$)$, then there is always another map $\varphi^{\prime}$, derived from $\varphi$, such that $\varphi^{\prime}$ satisfies both 1 ) and 2). Further, it is apparentily unknown whethex one enlarges the class of euclidean integral domains by enlarging $N$ to a well-ordered set of arbitrary cardinality, but this question will not concern us here except to say that whenever $A$ has finite residue classes; i.e., $A$ modulo any nonzero ideal is finite, then insisting on $N$ as a set of values is no restriction. We refer the reader to an excellent paper by $P$. Samuel [8] in which all of the above and much more is exposed with great clarity.

Let $A$ be as above. We define subsets $A_{n}$ of $A$ for $n \in N$ by induction as follows: $A_{0}=\{0\}$ and if $n \geqslant 1$, then $A_{n}^{\prime}=\bigcup_{a<n} A_{\alpha}$. Finally $A_{n}=\{b \in A\}$ there is a representative in $A_{n}^{\prime}$ of every residue class of $A$ modulo $\left.b A\right\}$. Setting $A_{:}^{\prime}=\bigcup_{n \in N} A_{n}, A$ is euclidean if and only if $A^{\prime}=A$ (see Motzlkin [6]). Further when $A^{\prime}=A$ we get a map $\varphi: A-\{0\} \rightarrow N$, where if $x \in A-$ $-\{0\}$ then there exists a mucque $n \geqslant 0$ such that $\infty \in A_{n+1}-A_{n}$ and $\varphi(x)=n$. Now not only does $\phi$ satisfy conditions 1) and 2) above, but if. $\phi^{\prime}$ is any other map satiafying condition 2$)$, then $\phi(x) \leqslant \varphi^{\prime}(x)$ for all $x \in A-\{0\}$. Hence Motakin justifiably ealls of the minimal algorithm for $A$.

Let $F$ be a global field, so $F$ is a finite extension of the rational numbers $Q$ or $F^{\prime}$ is a function field of one variable over a tinite field. Let $S$ be a nonempty finite set of prime divisors of $F$ such that $S$ contains all infinite (i.e. archimedean) prime divisors. For each finite (i.e. non-archimedean) 